\title{
Metasurface-based compact light engine for AR headsets
}

Seyedeh Mahsa Kamali, Ehsan Arbabi, Andrei Faraon

Seyedeh Mahsa Kamali, Ehsan Arbabi, Andrei Faraon, "Metasurface-based compact light engine for AR headsets," Proc. SPIE 11040, Optical Design Challenge 2019, 1104002 (27 February 2019); doi: 10.1117/12.2523720

SPIE Event: SPIE Optical Design Challenge, 2019, San Francisco, California, United States 


\title{
Metasurface-based compact light engine for AR headsets
}

\author{
Seyedeh Mahsa Kamali ${ }^{\mathrm{a}}$, Ehsan Arbabi $^{\mathrm{a}}$, and Andrei Faraon ${ }^{\mathrm{a}}$ \\ ${ }^{a}$ T. J. Watson Laboratory of Applied Physics and Kavli Nanoscience Institute, California \\ Institute of Technology, 1200 E California Blvd., Pasadena, CA 91125, USA
}

\begin{abstract}
Despite the great advances, potentials of augmented reality to fundamentally transform the way people use computers is partially hindered by the size and weight of the AR headsets. In waveguide-based devices, the light engine constitutes a significant portion of the total volume and weight. Dielectric metasurfaces have in recent years been used to demonstrate various high performance optical elements like blazed gratings and wide field of view lenses with small thicknesses, high efficiencies, and little stray light. Here, we report our work on the design of a compact light engine based on multi-metasurface optics with wide fields of view, integrated with three monochrome $\mu$-LED displays for red, green, and blue. The metasurfaces image the $\mu$-LEDs on the prism or grating couplers. This design avoids an important shortcoming of $\mu$-LEDs and metasurface lenses, i.e., each work well for a single wavelength. As an example, we present a design for $532 \mathrm{~nm}$, with over 3000 resolved angular points in an 8-mm-diameter field of view, and a total volume less than $0.65 \mathrm{cc}(<2 \mathrm{cc}$ for the three wavelengths). Limited by the total internal reflection region inside a waveguide with a 1.78 refractive index, the light engine can produce an image with over $1500 \times 1500$ points over a field of view slightly larger than $85^{\circ} \times 85^{\circ}$ in air. To the best of our knowledge, this is the first proposal and demonstration of such a system and therefore opens the path towards exploring the potentials of the metasurface diffractive optics technology for compact AR headsets with enhanced optical capabilities.
\end{abstract}

Keywords: Compact AR headset, Compact head moundet displays, Minitaurized light engine for AR headsets

\section{INTRODUCTION}

Powered by the great advancements in electronics, computer science, and compact refractive and diffractive micro-optics, ${ }^{1}$ headmounted AR displays have in recent years become a new category of consumer electronics. With many great proposals and demonstrations based on various technologies including visor and waveguide based systems ${ }^{2-5}$ many products from different companies are now hitting the market. Among various designs, waveguide-based designs seem more suitable for compact AR headsets with wide fields of view. In these devices, the light engine constitutes a significant portion of the volume and weight of the device, and can even limit its performance by not providing the whole field of view and resolution supported by the waveguide optics.

Here we present our work on the proposal and design of a compact light engine based on three monochrome $\mu$-LED displays imaged using multi-metasurface optical systems corrected for wide fields of view. Since each $\mu$-LED display has its own metasurface optics, this design significantly avoids the main challenge facing both $\mu$-LEDs and metasurfaces, that is, they each work well when designed for a single color. This shortcoming has so far prevented the use of $\mu$-LEDs in AR headsets because the pixel size is significantly increased when $\mu$-LEDs of different colors are combined to provide full RGB coverage on the same chip. On the other hand, hindered by the size and weight of optical elements, it has not been practical to use three separate $\mu$-LED displays as each of them would require its own imaging optics.

The proposed design overcomes this issue since the imaging optics for each color can be separate, while keeping the total volume and weight of the optics low (i.e., to lower than 2 cubic centimeters and less than 3

Further author information: (Send correspondence to A.F.)

S.K.: E-mail: skamali@caltech.edu

E.A.: E-mail: earbabi@caltech.edu

A.F.: E-mail: faraon@caltech.edu

Optical Design Challenge 2019, edited by Bernard C. Kress, Christophe Peroz, Proc. of SPIE Vol. 11040, 1104002 - () 2019 SPIE · CCC code: 0277-786X/19/\$18 · doi: 10.1117/12.2523720 
grams for the optics of the three colors combined). More specifically, we demonstrate a five layer metasurface design with a corrected field of view of $8 \mathrm{~mm}$ (close to $90^{\circ}$ inside a glass with a refractive index of 1.78) to provide near diffraction limited focusing with about 3000 resolved points. The optics has a collection NA of 0.25 , and delivers the collimated beams with RMS wavefront errors lower than 0.25 across its field to an aperture with a diameter of $2.1 \mathrm{~mm}$. Capped by the total internal reflection region of the waveguide (i.e., $35^{\circ}-80^{\circ}$, for an index of 1.78) the optics can deliver over $1500 \times 1500$ points for each color, within a field of view just above $85^{\circ} \times 85^{\circ}$ in air. We also present metasurface designs based on crystalline silicon nano-scatterers that can implement the proposed metasurfaces at all colors of interest $(480 \mathrm{~nm}, 532 \mathrm{~nm}$, and $635 \mathrm{~nm})$. In the end, we discuss the main challenges faced by this technology including the chromatic dispersion and point potential methods of mitigation. While the concept of metasurfaces has previously been proposed for use in AR devices, ${ }^{6,7}$ it has generally been limited to their application as periodic grating couplers. Being the first proposal and demonstration of its kind, we believe that this work will pave the way and encourage the exploration of potentials of metasurface optical elements for integration into compact optical systems used in AR headsets.

\section{CONCEPT}

Figure 1 schematically shows the concept of the metasurface based light engine AR display. As shown in Fig. $1 \mathbf{a}$, light from monochrome $\mu$-LED displays is collimated and directed to the waveguide, using multi-metasurface optical systems. Based on whether the eye box expanders and the out-coupling gratings can be combined for different colors or not (e.g., using volumetric Bragg gratings), one, two, or three waveguides might be used in the actual design. Here, for the sake of simplicity, we assume that the waveguides can be combined and therefore shown only one waveguide. As shown in Fig. 1b, the light engines for the three colors combined can be as small as 3 cubic centimeters (with about $2 \mathrm{~mm}$ of thickness reserved for the $\mu$-LED displays and their electrical circuitry). Figure 1c schematically shows a zoomed-in view of the light engine for the red color $\mu$-LED display. The multiple metasurface system images each pixel of the $\mu$-LED display into the grating coupler aperture with a corrected wavefront. To better visualize the compactness of the proposed platform, a schematic illustration of the whole metasurface-based light engine mounted on a typical pair of glasses is shown in Fig 1d.

\section{METASURFACE DESIGN}

In general, a metasurface consists of an array of nano-scatterers that control the phase, polarization, and amplitude of light as it passes through them. This is schematically shown in Fig. 2, where the outgoing light has a phase and polarization that is locally controlled by the metasurface. While metasurfaces are more than a few decades old, ${ }^{8}$ the advancements in theoretical and computational electromagnetics, ${ }^{9-11}$ micro and nanofabrication techniques, and their widespread availability have in recent years resulted in a greatly increased interest in this field. ${ }^{8,12,13}$ High contrast dielectric metasurfaces, are a specific case in which the nano-scatterers are made of high-index dielectrics (such as silicon, titanium dioxide, silicon nitride, etc.) and are surrounded by low index matrices. In general, the nano-scatterers can either be patterned and fabricated on a planar substrate [Fig. 2a], or transferred to a flexible substrate and conformed to a refractive object with a non-planar surface [Fig. 2b], forming a conformal optical metasurface. In addition to changing the optical function of the refractive element, the combined conformal metasurface-object optical system can perform optical functions that neither of its components can do alone.

A widely used category of high contrast dielectric metasurfaces is based on dielectric nano-scatterers (which we call nano-posts from here on) with a given set of cross sections (e.g., square, circular, rectangular, etc.) and different lateral dimensions, such as the one shown in Fig. 3a. In these structures, the nano-posts can be treated as multi-mode truncated waveguides with multiple resonances, the interplay of which determines the scattering properties of the nano-posts. ${ }^{14}$ In order to better suppress higher order diffractions and unwanted scattering, the nano-posts are generally patterned on a uniform lattice, for instance a square lattice like Fig. $3 \mathbf{b}$. In addition, the lattice should be subwavelength and satisfy the Nyquist sampling rate for the used material systems and transmission functions that are implemented. ${ }^{15}$ With proper design, the nano-post array can have very high transmission efficiencies (80\%-100\%, depending on the material losses), while at the same time providing full $0-2 \pi$ phase coverage with changing the nano-post dimensions. ${ }^{16-18}$ Figure $3 \mathbf{c}$ shows the transmission phases and amplitudes for three arrays with different lattice constants and nano-post thicknesses designed for blue, green, 


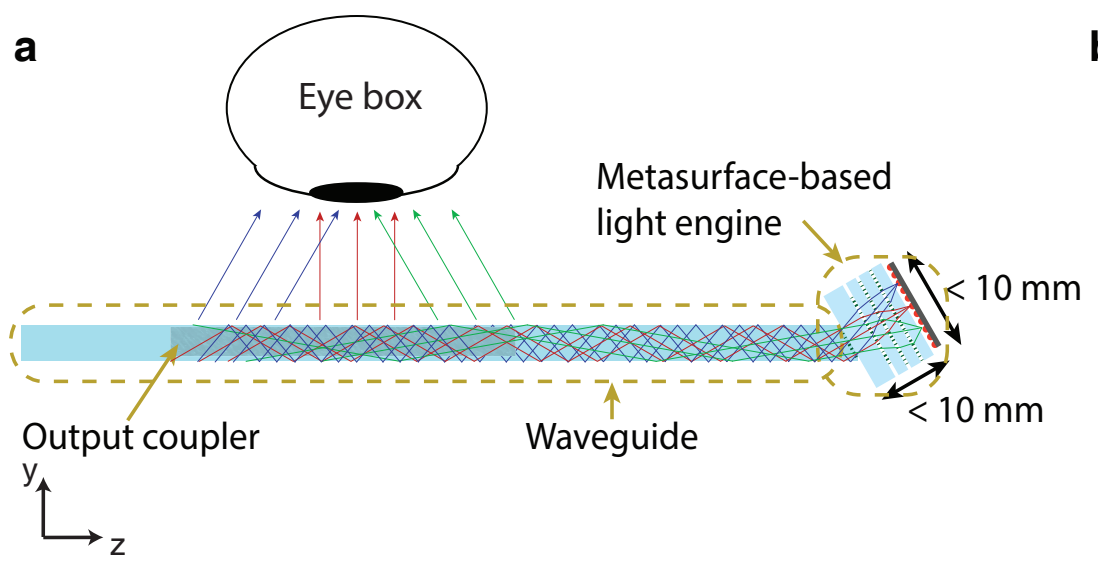

C
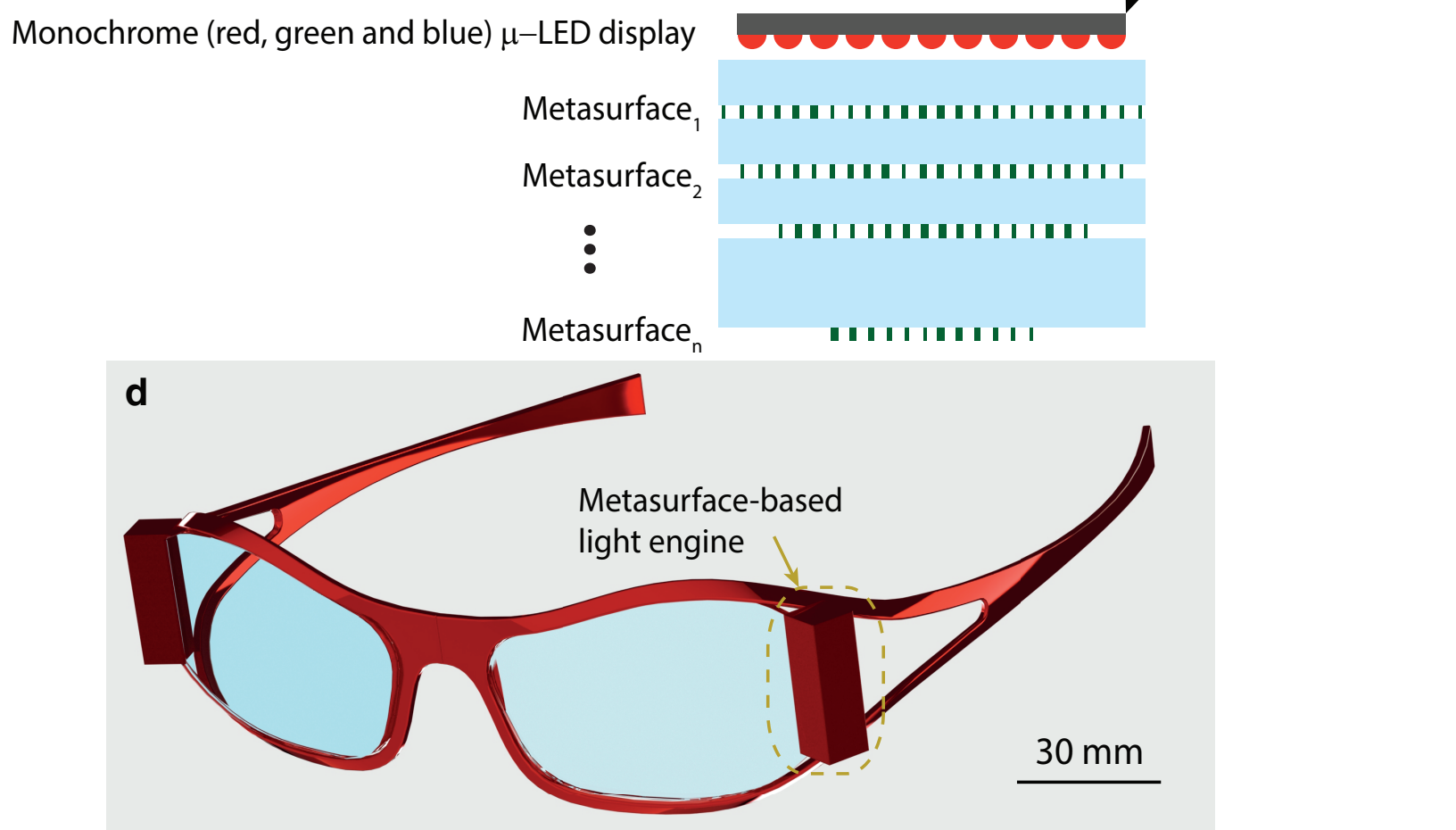

Figure 1. Concept of $\mu$-LED and metasurface-based light engines for AR glasses based on waveguides. (a) Schematic topview of the glass, showing the relative locations of the waveguide, light engine, and the eye box. The metasurface-based optics collimates the light from the monochrome $\mu$-LED displays and directs it to the prism coupler. Inside the waveguide, light is expanded by the eye box expander and finally out-coupled using holographic volume gratings that could increase the field of view. (b) Schematics of the side view of the $\mu$-LED displays and the metasurface based optics, showing all three colors at the same time. The whole light engine is smaller than $30 \mathrm{~mm} \times 10 \mathrm{~mm} \times 10 \mathrm{~mm}$, with the total volume of the optics smaller than 2 cubic centimeters. All three colors can either share the same waveguide, or there maybe more than one waveguide, each for one or two of the colors depending on the possibility of combining the eye box expanders and out-coupling gratings. (c) Zoomed-in view of the $\mu$-LED display and typical metasurfaces forming the light engine optics for the red color. (d) Schematic illustration of the whole metasurface-based light engine mounted on a typical pair of glasses. 
a

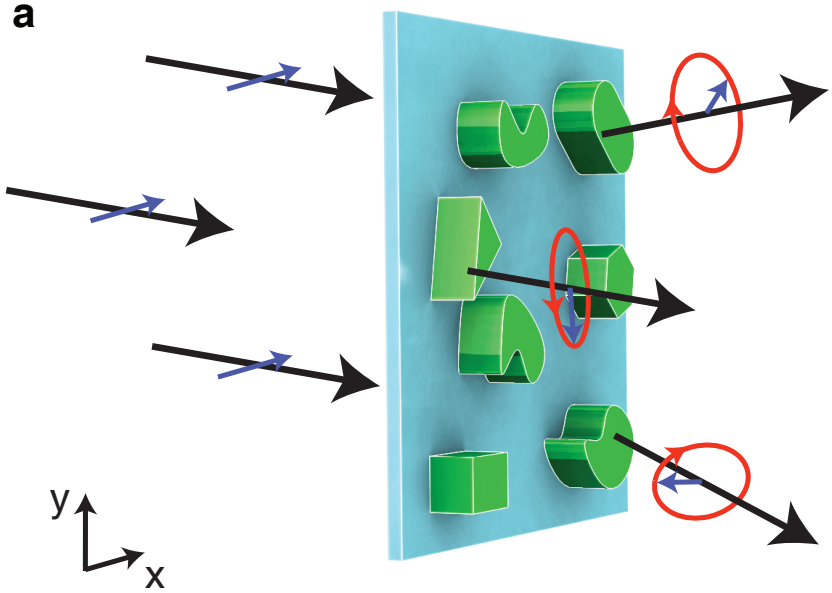

b

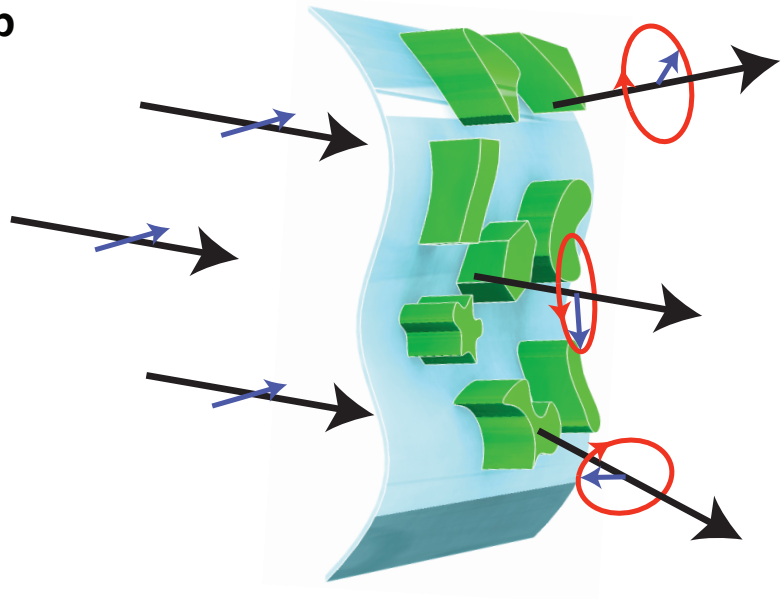

Figure 2. Schematic demonstration of planar and conformal metasurfaces. (a) A flat metasurface is in general composed of nano-scatterers that are designed to scatter incident light to an outgoing light with arbitrary phase, polarization, and amplitude. In particular, dielectric metaurfaces that are composed of high-index dielectric nano-scatterers can control the phase and polarization with high efficiency and low stray light for large phase gradients (equivalent to high numerical apertures). (b) Conceptual demonstration of a conformal metasurface, where the high-index dielectric nano-scatterers are transferred to a flexible substrate and conformed to a surface with an arbitrary shape. The combined element can perform optical functions that neither of the components (i.e., the refractive element and the flat metasurface) could provide on their own.

and red $(480 \mathrm{~nm}, 532 \mathrm{~nm}$, and $635 \mathrm{~nm}$, respectively). In order to use the same material system for all colors, all metasurfaces are designed using crystalline silicon (c-Si). This way, the metasurfaces for all three colors can be fabricated on the same substrates, increasing the mechanical robustness and decreasing the required packaging volume and wight significantly. The three graphs in Fig. 3c show the clear ability of the designed nano-post arrays to provide full phase coverage with high transmission. One important property of this type of metasurface is the very low coupling between adjacent nano-posts as the the high index contrast between the nano-posts and the surrounding media results in almost all of the optical energy being confined inside the nano-posts. ${ }^{16,17}$ As a result, sizes of the nano-posts can be changed without significantly altering the transmission phases and amplitudes of its neighbors. This enables high efficiency beam deflection to large angles, which in turn results in thin high-efficiency lenses with large numerical apertures. ${ }^{17,19}$

\section{LIGHT ENGINE OPTICS DESIGN}

While a single-layer metasurface lens has the ability to focus light to a point without spherical aberrations, ${ }^{20,21}$ multiple metasurface layers are required in order to correct for other monochromatic aberrations. ${ }^{22,23}$ Figure 4 summarizes the results of one such design, where 5 metasurface layers are cascaded to provide near-diffraction limited imaging of the green $\mu$-LED to the input aperture of the waveguide (i.e., the prism edge). As seen in Fig. $4 \mathbf{a}$, the first four metasurfaces have radii of $4.5 \mathrm{~mm}$, and are each separated $1 \mathrm{~mm}$ from the previous surface with separating glasses that each have a refractive index of $\sim 1.5$. The fifth glass layer is $4 \mathrm{~mm}$ thick and has an index of $\sim 1.78$, to match that of the waveguide. The fifth metasurface has a diameter of $2.1 \mathrm{~mm}$, resulting in an input aperture of $2.1 \mathrm{~mm}$ at the coupling prism. The total thickness of the device is $8 \mathrm{~mm}$, and it could be fitted inside a $9 \mathrm{~mm} \times 9 \mathrm{~mm} \times 8 \mathrm{~mm}$ cube, and thus it will occupy a volume of less than 0.65 cubic centimeters (i.e., $<2$ cubic centimeters for the three wavelengths). In addition, the total glass volume is about $3 \times 0.4=1.2$ cubic centimeters, so the total weight of the glass would be about 3 grams (assuming a density of 2.5 grams $/ \mathrm{cc}$ ).

As seen from the spot diagram simulations of Fig. $4 \mathbf{b}$, the system has a near-diffraction limited focusing up to a field of view of $4 \mathrm{~mm}$, corresponding to an angle of $\sim 44$ degrees $(0.77 \mathrm{Rad})$. Considering the $\sim 1.5 \mathrm{Rad}$ field of view, the optics has more than 3000 resolvable points along the diagonal (corresponding to more than $2000 \times 2000$ points). The light at the object plane is telecentric and is collected up to a numerical aperture of 0.25 for most of the surface, except very close to $4 \mathrm{~mm}$ where the effective collection NA is slightly less. The 
optimized phase profiles for the five metasurfaces are plotted in Fig. 4c. It is worth noting that for two of the metasurfaces the maximum required OPD is about 5000 waves, which corresponds to a thickness of more than $3 \mathrm{~mm}$ (assuming a glass with an index of 1.78). Figure $4 \mathbf{d}$ schematically shows the light engine integrated with a waveguide that has a prism with a 57.5-degree edge, and therefore a thickness just below $1.8 \mathrm{~mm}$. Assuming a waveguide index of 1.78 (similar to the one used here), angles from 35 to 80 degrees can be used for coupling as they are bounded by total internal reflection inside the waveguide. This means that an angular area of $45^{\circ} \times 45^{\circ}$ can be directly coupled inside the waveguide. Given the smaller than $0.5 \mathrm{mRad}$ angular resolution, this corresponds to a resolution of more than $1500 \times 1500$ points for each color. With the three wavelengths combined, this means a total of more than 7 megapixels. On the $\mu$-LED display, this roughly corresponds to a field of view of $4 \mathrm{~mm} \times 4 \mathrm{~mm}$. Given that $\mu$-LED displays with pixel sizes of about $3 \mu \mathrm{m}$ have already been demonstrated, ${ }^{24,25}$ it seems reasonable to think that ones with pixel pitches of about $2.5 \mu \mathrm{m}$ are feasible too. We should also note here that for such a smaller field of view, the metasurface optics can also have smaller diameter (about $7 \mathrm{~mm}$ ) and smaller total volume (about $1.2 \mathrm{cc}$ for the three wavelengths combined). In addition, the $45^{\circ} \times 45^{\circ}$ field of view inside the waveguide translates to a larger than $85^{\circ} \times 85^{\circ}$ field of view in air.
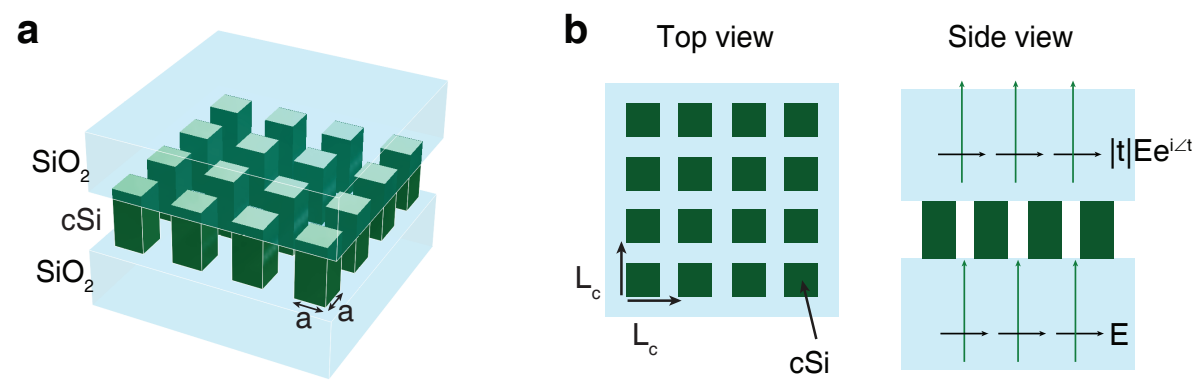

C
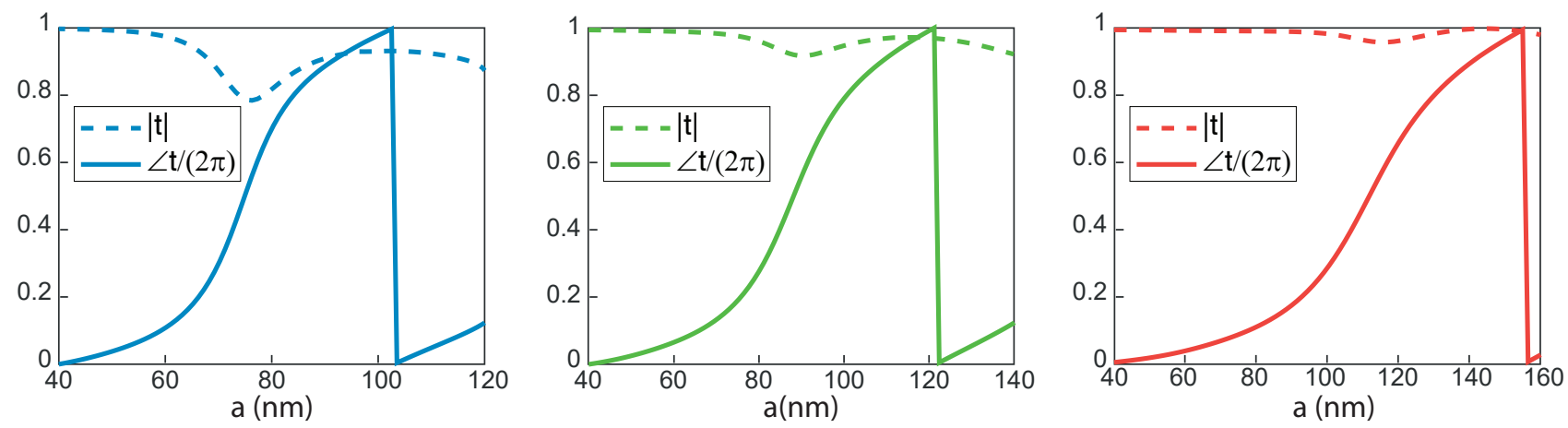

Figure 3. Schematics and design graphs for dielectric metasurfaces based on c-Si. (a) Schematic illustration of the proposed metasurface structure that is composed of c-Si nano-posts with square cross sections, sandwiched between two low-index substrates. For fabrication considerations, one of the substrates can actually be fabricated by spin coating a thick spin-on glass or polymer layer that does not fill in the nano-post gaps. (b) Top and side views of the proposed metasurface structure, showing the lattice constant $L_{c}$, and the complex transmission $t$. The optimal metasurface structure will have different thicknesses and lattice constants for the three different colors. (c) The simulated transmission amplitudes and phases for metasurfaces designed for blue (left, $480 \mathrm{~nm}$ ), green (middle, $532 \mathrm{~nm}$ ), and red (right, $635 \mathrm{~nm}$ ). The metasurfaces have thicknesses of $224 \mathrm{~nm}, 270 \mathrm{~nm}$, and $340 \mathrm{~nm}$, and lattice constants of $180 \mathrm{~nm}, 200 \mathrm{~nm}$, and $240 \mathrm{~nm}$ for blue, green, and red, respectively. Measured refractive indices of c-Si on quartz was used for the simulations. The used index values are $4.413-0.04033 j, 4.136-0.01027 j$, and $3.874-0.008432 j$ at wavelengths of $480 \mathrm{~nm}, 532 \mathrm{~nm}$, and $635 \mathrm{~nm}$, respectively. The graphs ensure full $2 \pi$ phase coverage at all required wavelengths with high transmission. In addition, the high refractive index of c-Si ensures the ability to optimize metasurfaces for large deflection angles with high diffraction efficiencies.

Figures $4 \mathbf{e}$ and $4 \mathbf{f}$ show the first nonzero standard Zernike coefficients $\left(\mathrm{Z}_{4}\right.$ to $\left.\mathrm{Z}_{1} 4\right)$ and the field curvature, respectively. As seen from both graphs, the dominant aberration is astigmatism. Nevertheless, the RMS wavefront error (calculated from rays in reference to the centroid) is smaller than 0.25 waves over the whole field of 


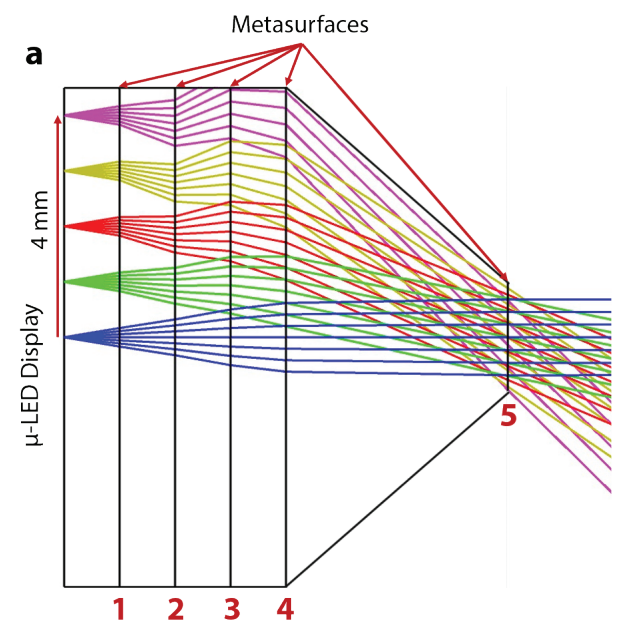

b
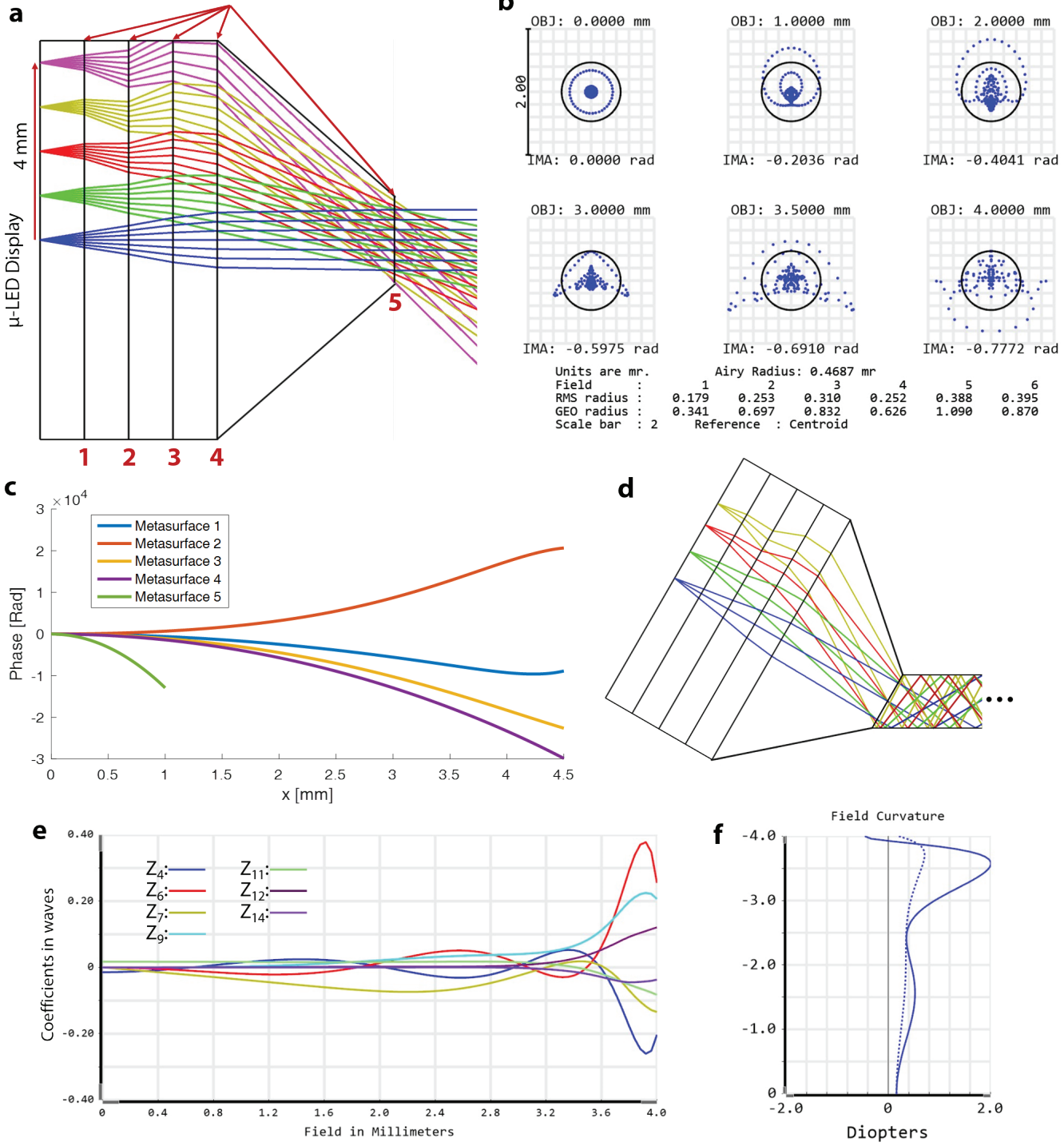

Figure 4. Ray tracing simulation results of a metasurface based design composed of five layers. (a) A five layer metasurface design with a field of view of $8 \mathrm{~mm}$ (diameter) and collection NA of 0.25 (telecentric). Locations of the metasurfaces are denoted with the numbered vertical lines. The system includes 4 glass layers of $1 \mathrm{~mm}$ thickness each, and a fifth layer that is $4 \mathrm{~mm}$ thick (the conical layer). The first 4 layers have refractive indices of $\sim 1.5$, and the fifth layer has an index of $\sim 1.78$ to match that of the prism and the waveguide. The first 4 metasurfaces have radii of $4.5 \mathrm{~mm}$, and the fifth one has a radius of $1.05 \mathrm{~mm}$, resulting in a coupling aperture diameter of $2.1 \mathrm{~mm}$. (b) Spot diagram simulations for six different points in the field of view, showing close to diffraction limited behavior over the field. Given the angular field of view of $>1.5 \mathrm{Rad}$, and the diffraction limited Airy radius of $<0.5 \mathrm{mRad}$, there are more than 3000 resolvable angles along the diameter. (c) Optimized phase profiles for the five metasurfaces, numbered from left to the right according to the panel a. It is worth noting that the maximum phases are equivalent to about 5000 waves, or a thickness of $\sim 3 \mathrm{~mm}$. (d) Schematics of the light engine mounted on a 57.5-degree prism-coupled waveguide. The last point of the field (i.e., yellow color) denotes $2.8 \mathrm{~mm}$, corresponding to the half width of a square $\mu$-LED display with a diagonal of $8 \mathrm{~mm}$ (thus a $4 \mathrm{~mm}$ field of view). (e) Nonzero standard Zernike coefficients 4-14 versus the field of view, calculated for an aperture diameter of $2.1 \mathrm{~mm}$. The RMS wavefront error (referenced to centroid and calculated from rays) is smaller than 0.25 waves over the entire field of view, and is significantly below 0.25 waves over most of the field. (f) The field curvature versus field of view, showing less than 2 diopters of curvature, and also showing that the dominant aberration is astigmatism. 
view, and is smaller than 0.1 waves for field values below $3 \mathrm{~mm}$. Calculated from the Zernike coefficients, the wavefront error peaks at slightly above 0.4 waves close to $3.9 \mathrm{~mm}$, and is smaller than 0.25 waves below $3 \mathrm{~mm}$.

\section{DISCUSSION}

Like any other optical system, the design of the metasurface light engine optics involves various compromises. For instance, the collection NA and the angular resolution of the optics can be increased at the expense of field of view (with the same volume constraints). As a result, other designs that are closer to the perfect human eye resolution of $0.3 \mathrm{mRad}$ are possible with a field of view smaller than $8 \mathrm{~mm}$ diagonal. In addition, the number of metasurface layers is inversely proportional to the system efficiency, while it is possible to increase the collection NA with more layers. Therefore, a compromise should be made that maximizes the total system efficiency in this regard as well.

The main drawback of using metasurface optics is their large chromatic dispersion, which unlike refractive optics does not mainly originate from material dispersion and as such, unlike the case of refractive elements, ${ }^{26}$ it cannot be solved using combination of different materials. While it is possible (and in fact relatively straight forward) to design metasurfaces that have high diffraction efficiencies over bandwidths of more than $10 \%,{ }^{27,28}$ similar to other diffractive devices, ${ }^{29}$ metasurfaces, have a large negative dispersion resulting from a phase profile that is almost constant with wavelength. ${ }^{30}$ As a result, the regular chromatic dispersion of the metasurfaces can significantly decrease the achievable resolution and degrade the device performance. For instance, while the exact amount of degradation depends on the actual LED linewidth, for a typical value of 30-40 nm the resolution might drop by more than a factor of three. Despite the modest success of the independent group delay-phase delay control method in addressing the chromatic dispersion issue in metasurfaces, ${ }^{31-33}$ its applicability is severely limited to devices with miniature sizes (i.e., a couple of hundred microns in diameter) because of the requirement for extremely high quality factor resonances in millimeter scale devices. As a result, the two main approaches for addressing the chromatic dispersion issue are using metasurface diffractive/refractive combinations, ${ }^{1,34,35}$ and decreasing the emission bandwidth of $\mu$-LEDs. With these methods, it might be possible to decrease the effect of chromatic dispersion to levels that are acceptable for human eye.

The unique properties of metasurfaces enable a few platforms for tunable, conformal, and folded optical elements and systems that are more difficult to achieve with other technologies. For instance, various types of tunable metasurface devices, either based on stretchable substrates ${ }^{15,36}$ or micro-electro-mechanically tunable doublets $^{37}$ have been recently demonstrated that can potentially be integrated into the light engine optics to enable time-multiplexed multi-focus image rendering. Obviously, other types of flat tunable lenses, such as liquid crystal ones ${ }^{38}$ can also be used for this purpose. Conformal metasurfaces ${ }^{39,40}$ provide additional design degrees of freedom by allowing for the metasurface to be non-planar. In addition to higher diffraction efficiencies and lower stray light, one of their main advantages over their conventional Fresnel counterparts is that the metasurface phase profile does not need to have the same symmetries of the underlying surface since the phase profile is defined lithographically in a different step. Finally, the folded metasurface optical platform can be used to reduce the total volume of the optics by using the metasurface substrate multiple times as propagation space. ${ }^{27}$ While requiring more complicated design strategies (and potentially having a poorer optical quality), the folded metasurface platform can result in more compact devices with less complicated fabrication steps as most of the alignment steps can be eliminated.

Finally, while the use of low-throughput high-end patterning techniques like electron beam lithography is possible for proof of concept demonstrations and design rounds, it is essential to use low-cost high-throughput techniques such as nano-imprint lithography ${ }^{41,42}$ for mass production. Using such techniques and material systems like silicon for which high-quality etching processes are available can potentially reduce the fabrication cost of the optics.

\section{CONCLUSION}

In this manuscript we have proposed and demonstrated a design for a compact light engine composed of three monochrome $\mu$-LED displays imaged using multi-metasurface optical systems corrected over a wide field of view. This design avoids the main challenge faced by both small $\mu$-LED displays and metasurfaces, i.e., their operation 
is limited to one color range. As a proof of principle, we designed a five-layer metasurface optical system with a corrected field of view of $8 \mathrm{~mm}$ diagonal (close to $90^{\circ}$ angular inside a glass with a refractive index of 1.78), with a close to diffraction limited operation over the whole field of view. With a total size smaller than 2 cubic centimeters, the metasurface optics has the ability to generate $2000 \times 2000$ diffraction limited points within its field of view. The same optics can be coupled to a waveguide delivery system and provide a wide field of view of $>85^{\circ} \times 85^{\circ}$ in air. While several fundamental and technical challenges such as the chromatic dispersion, alignment of different colors, and fabrication tolerances need to be investigated, we believe that this work demonstrates a clear potential for metasurfaces to address some of the challenges faced in the design of compact light engines for headmounted AR displays.

\section{REFERENCES}

[1] Kress, B. C. and Meyrueis, P., [Applied Digital Optics: From MicroOptics to Nanophotonics], John Wiley Sons, Ltd (2009).

[2] Kress, B. and Starner, T., "A review of head-mounted displays (hmd) technologies and applications for consumer electronics," in [SPIE Defense, Security, and Sensing], 8720, 13, SPIE (2013).

[3] $\mathrm{Hu}, \mathrm{X}$. and Carollo, J., "Head mounted display device with multiple segment display and optics," USPatentApp. (14/923,144) (2017).

[4] Travis, A., Kollin, J. S., and Georgiou, A., "Virtual image display with curved light path," USPatent $(9,759,919)(2017)$.

[5] Maimone, A., Georgiou, A., and Kollin, J. S., "Holographic near-eye displays for virtual and augmented reality," ACM Trans. Graph. 36(4), 1-16 (2017).

[6] Lin, D., Melli, M., Poliakov, E., Hilaire, P. S., Dhuey, S., Peroz, C., Cabrini, S., Brongersma, M., and Klug, M., "Optical metasurfaces for high angle steering at visible wavelengths," Sci. Rep. 7(1), 2286 (2017).

[7] Shi, Z., Chen, W. T., and Capasso, F., "Wide field-of-view waveguide displays enabled by polarizationdependent metagratings," in [SPIE Photonics Europe], 10676, 6, SPIE (2018).

[8] Lalanne, P. and Chavel, P., "Metalenses at visible wavelengths: past, present, perspectives," Laser Photon. Rev. 11(3), 1600295 (2017).

[9] Oskooi, A. F., Roundy, D., Ibanescu, M., Bermel, P., Joannopoulos, J. D., and Johnson, S. G., "Meep: A flexible free-software package for electromagnetic simulations by the FDTD method," Comput. Phys. Commun. 181(3), 687-702 (2010).

[10] Liu, V. and Fan, S., "S4 : A free electromagnetic solver for layered periodic structures," Comput. Phys. Commun. 183(10), 2233-2244 (2012).

[11] Zhang, S., Wyrowski, F., and Tervo, J., "Efficient grating simulation for general incident beam," in [SPIE MOEMS-MEMS], 8977, 12, SPIE (2014).

[12] Kamali, S. M., Arbabi, E., Arbabi, A., and Faraon, A., "A review of dielectric optical metasurfaces for wavefront control," Nanophotonics 7(6), 1041-1068 (2018).

[13] Qiao, P., Yang, W., and Chang-Hasnain, C. J., "Recent advances in high-contrast metastructures, metasurfaces, and photonic crystals," Adv. Opt. Photonics 10(1), 180-245 (2018).

[14] Lalanne, P., "Waveguiding in blazed-binary diffractive elements," J. Opt. Soc. Am. A 16(10), 2517-2520 (1999).

[15] Kamali, S. M., Arbabi, E., Arbabi, A., Horie, Y., and Faraon, A., "Highly tunable elastic dielectric metasurface lenses," Laser Photon. Rev. 10(6), 1062-1062 (2016).

[16] Lalanne, P., Astilean, S., Chavel, P., Cambril, E., and Launois, H., "Blazed binary subwavelength gratings with efficiencies larger than those of conventional échelette gratings," Opt. Lett. 23(14), 1081-1083 (1998).

[17] Arbabi, A., Horie, Y., Ball, A. J., Bagheri, M., and Faraon, A., "Subwavelength-thick lenses with high numerical apertures and large efficiency based on high-contrast transmitarrays," Nat. Commun. 6(7069), 7069 (2015).

[18] Vo, S., Fattal, D., Sorin, W. V., Zhen, P., Tho, T., Fiorentino, M., and Beausoleil, R. G., "Sub-wavelength grating lenses with a twist," IEEE Photon. Technol. Lett. 26(13), 1375-1378 (2014).

[19] Lin, D., Fan, P., Hasman, E., and Brongersma, M. L., "Dielectric gradient metasurface optical elements," Science 345(6194), 298-302 (2014). 
[20] Aieta, F., Genevet, P., Kats, M. A., Yu, N., Blanchard, R., Gaburro, Z., and Capasso, F., "Aberration-free ultrathin flat lenses and axicons at telecom wavelengths based on plasmonic metasurfaces," Nano Lett. 12(9), 4932-4936 (2012).

[21] Arbabi, E., Li, J., Hutchins, R. J., Kamali, S. M., Arbabi, A., Horie, Y., Van Dorpe, P., Gradinaru, V., Wagenaar, D. A., and Faraon, A., "Two-photon microscopy with a double-wavelength metasurface objective lens," Nano Lett. 18(8), 4943-4948 (2018).

[22] Buralli, D. A. and Morris, G. M., "Design of a wide field diffractive landscape lens," Appl. Opt. 28(18), 3950-3959 (1989).

[23] Arbabi, A., Arbabi, E., Kamali, S. M., Horie, Y., Han, S., and Faraon, A., "Miniature optical planar camera based on a wide-angle metasurface doublet corrected for monochromatic aberrations," Nat. Commun. 7(13682), 13682 (2016).

[24] "http : //www.eenewseurope.com/news/leti - solves - stacking - issue - micro - led - displays," (accessdate : 01/22/2019).

[25] "https://www.ledinside.com/news/2018/3/jbd_devises_new_micro_led_technology_to_make_ultra_compact_ micro_led_microdisplays," (accessdate : 01/22/2019).

[26] Unger, B. L. and Ramsey, J. L., "Athermalized and achromatized multispectral optical systems and methods of designing same," USPatentApp. (15/689,770) (2018).

[27] Faraji-Dana, M., Arbabi, E., Arbabi, A., Kamali, S. M., Kwon, H., and Faraon, A., "Compact folded metasurface spectrometer," Nat. Commun. 9(1), 4196 (2018).

[28] Wang, L., Kruk, S., Tang, H., Li, T., Kravchenko, I., Neshev, D. N., and Kivshar, Y. S., "Grayscale transparent metasurface holograms," Optica 3(12), 1504-1505 (2016).

[29] Faklis, D. and Morris, G. M., "Spectral properties of multiorder diffractive lenses," Appl. Opt. 34(14), 2462-2468 (1995).

[30] Arbabi, E., Arbabi, A., Kamali, S. M., Horie, Y., and Faraon, A., "Multiwavelength polarization-insensitive lenses based on dielectric metasurfaces with meta-molecules," Optica 3(6), 628-633 (2016).

[31] Arbabi, E., Arbabi, A., Kamali, S. M., Horie, Y., and Faraon, A., "Controlling the sign of chromatic dispersion in diffractive optics with dielectric metasurfaces," Optica 4(6), 625-632 (2017).

[32] Chen, W. T., Zhu, A. Y., Sanjeev, V., Khorasaninejad, M., Shi, Z., Lee, E., and Capasso, F., "A broadband achromatic metalens for focusing and imaging in the visible," Nat. Nanotechnol. 13, 220-226 (2018).

[33] Wang, S., Wu, P. C., Su, V.-C., Lai, Y.-C., Chen, M.-K., Kuo, H. Y., Chen, B. H., Chen, Y. H., Huang, T.-T., and Wang, J.-H. e. a., "A broadband achromatic metalens in the visible," Nat. Nanotechnol. 13, $227-232(2018)$.

[34] Miyamoto, K., "The phase fresnel lens," J. Opt. Soc. Am. 51(1), 17-20 (1961).

[35] Swanson, G. J., "Binary optics technology: the theory and design of multi-level diffractive optical elements," tech. rep., DTIC Document (1989).

[36] Ee, H.-S. and Agarwal, R., "Tunable metasurface and flat optical zoom lens on a stretchable substrate," Nano Lett. 16(4), 2818-2823 (2016).

[37] Arbabi, E., Arbabi, A., Kamali, S. M., Horie, Y., Faraji-Dana, M., and Faraon, A., "Mems-tunable dielectric metasurface lens," Nat. Commun. 9(1), 812 (2018).

[38] Jamali, A., Bryant, D., Zhang, Y., Grunnet-Jepsen, A., Bhowmik, A., and Bos, P. J., "Design of a large aperture tunable refractive fresnel liquid crystal lens," Appl. Opt. 57(7), B10-B19 (2018).

[39] Kamali, S. M., Arbabi, A., Arbabi, E., Horie, Y., and Faraon, A., "Decoupling optical function and geometrical form using conformal flexible dielectric metasurfaces," Nat. Commun. 7(11618), 11618 (2016).

[40] Burch, J. and Di Falco, A., "Surface topology specific metasurface holograms," ACS Photonics , Article ASAP (2018).

[41] Peroz, C., Chauveau, V., Barthel, E., and Sondergard, E., "Nanoimprint lithography on silica solgels: A simple route to sequential patterning," Adv. Mater. 21(5), 555-558 (2009).

[42] Pina-Hernandez, C., Lacatena, V., Calafiore, G., Dhuey, S., Kravtsov, K., Goltsov, A., Olynick, D., Yankov, V., Cabrini, S., and Peroz, C., "A route for fabricating printable photonic devices with sub-10 nm resolution," Nanotechnology 24(6), 065301 (2013). 